\title{
DIFICULTADES QUE DEMUESTRAN LOS ESTUDIANTES DE EDUCACIÓN BÁSICA EN LA INTERPRETACIÓN DE GRÁFICOS CINEMÁTICOS
}

\section{DIFFICULTIES DEMONSTRATED BY BASIC EDUCATION STUDENTS IN THE INTERPRETATION OF KINEMATIC GRAPHICS}

\author{
Raúl Prada Núñez ${ }^{1}$ \\ Audin Aloiso Gamboa Suárez² \\ William Rodrigo Avendaño Castro ${ }^{3}$
}

\section{RESUMEN}

Este documento presenta las dificultades que se identificaron en un grupo de 76 estudiantes de sexto a noveno grado de una institución educativa de naturaleza privada cuyo énfasis de formación es la enseñanza de las Ciencias Naturales. Se les aplicó un cuestionario en el

$1 \quad$ Magister en Educación Matemática. Docente Investigador Universidad Francisco de Paula Santander. E-mail: raulprada@ufps.edu.co, Orcid: https://orcid. org/0000-0001-6145-1786

2 Doctor en Ciencias de la Educación. Docente Investigador Universidad Francisco de Paula Santander. E-mail: audingamboa@ufps.edu.co, Orcid: https://orcid. org/0000-0001-9755-6408

3 Doctor en Ciencias Sociales y Humanas. Docente Investigador Universidad Francisco de Paula Santander. E-mail: williamavendano@ufps.edu.co, Orcid: https://orcid. org/0000-0002-7510-8222 que había cinco situaciones propuestas las cuales se fundamentaban en el gráfico PosiciónTiempo asociado al Movimiento Rectilíneo Uniforme. Se adopta un enfoque cuantitativo a nivel descriptivo con un diseño de campo, puesto que el cuestionario fue diligenciado por los informantes primarios. Se identificó como principal descubrimiento la presencia de concepciones alrededor del tema para no existe comprensión total del tema a causa de la metodología de enseñanza promocionada por el docente. Los resultados muestran que los estudiantes tienen una larga lista de dificultades principalmente por la falta de articulación de los conceptos matemáticos en contextos físicos, situación que les impide analizar los enunciados 
propuestos, razonar sobre los planteamientos y mejorar en el proceso de búsqueda de alternativas de solución con argumentos claros.

PALABRAS CLAVE: Registros semióticos de representación, concepciones, gráficas de espacio-tiempo.

\section{ABSTRACT}

This document presents the difficulties that were identified in a group of 76 students from sixth to ninth grade of a private educational institution whose training emphasis is the teaching of Natural Sciences. A questionnaire was applied to them in which there were five proposed situations which were based on the PositionTime graph associated with Uniform Rectilinear Motion. A quantitative approach was adopted at a descriptive level with a field design since the questionnaire was filled in by the primary informants. The main finding was identified as the presence of conceptions about the subject, so that there is no total understanding of the subject due to the teaching methodology promoted by the teacher. The results show that students have a long list of difficulties mainly due to the lack of articulation of mathematical concepts in physical contexts, a situation that prevents them from analyzing the proposed statements, reasoning about the approaches, and improving in the process of searching for alternative solutions with clear arguments.

KEYWORDS: Semiotic registers of representation, conceptions, space-time graphics.

\section{INTRODUCCIÓN}

La comunicación siempre se ha considerado una acción natural entre dos o más individuos en la naturaleza, independientemente del tipo de medio o lenguaje utilizado. En el contexto de la humanidad, la comunicación se muestra como un poderoso recurso que facilita el intercambio de ideas o mensajes para asegurar el entendimiento entre los actores involucrados.

A partir de esta inducción surge la pregunta, ¿dónde estaríamos como raza humana si no pudiéramos contar con la comunicación? Como se menciona en Vélez y Solórzano (2016) al definir la comunicación como "La acción de acercamiento y el intercambio de mensajes, es lo que hace que las personas se conozcan, dialoguen, interpreten las necesidades de los demás, lo que tienen y lo que requieren" ( $p$. 253), entonces este proceso es necesario en la interacción de las personas y cobra mayor importancia cuando se habla del proceso educativo. Dado que la educación ha sido históricamente un factor preponderante en el desarrollo de la sociedad, ya que de ella surgen ideas y posibles alternativas de solución a las dificultades que se presentan en la vida cotidiana. La comunicación educativa exige el compromiso de todos los implicados (estudiantes, docentes, directivos, padres y comunidad en general), pero en lo que afecta directamente a la calidad de la educación, hay que destacar que debe existir una buena comunicación entre el docente y los estudiantes para garantizar que éstos comprendan los mensajes que el docente quiere compartir. Sin olvidar la importancia de la comunicación no verbal expresada por el docente hacia los estudiantes, hacia sus necesidades y hacia el estudio de la materia que orienta (Domínguez, 2009).

Pero para garantizar la calidad de la comunicación educativa, se requiere que el docente asuma un rol protagónico, ya que antes de abordar los conceptos de la disciplina, primero debe estructurar didácticamente su clase, con el fin de despertar el interés y la motivación de sus estudiantes por el tema a desarrollar, llevándolos a sentirse confiados y seguros en clase y luego, propiciar espacios de reflexión donde prime el pensamiento crítico, el razonamiento, la argumentación, entre otros. Tal 
y como se recoge en la investigación de Enkvist (2010) al referirse a los resultados del informe McKinsey sobre los factores que influyen en los buenos resultados en la educación, en el que se destaca que "el factor clave en educación son los profesores. Lo importante no es tanto la inversión en edificio..., sino en la inteligencia y la preparación del profesor" (p. 50).

El contexto actual en el que viven los estudiantes se caracteriza por la fuerte tendencia a la globalización con el uso dominante de los recursos tecnológicos, lo que ha afectado el proceso educativo en la generación y demanda de nuevas competencias científicas para ser parte activa de la sociedad. Como se afirma, "el desarrollo de competencias asociadas al potencial formativo de las ciencias: capacidad crítica, reflexiva y analítica, conocimientos técnicos y habilidades, valoración del trabajo y capacidad para crear e investigar" (Castro \& Ramírez, 2013, p. 31).

Los artículos 5, 7, 9 y 13 de la Ley 115 (1994) establecen la enseñanza de las Ciencias Naturales como uno de los fines de la educación en Colombia. Posteriormente, a través de Ministerio de Educación Nacional (Mineducación, 1998), se expiden los Lineamientos Curriculares y los Estándares de Competencias con el fin de promover el desarrollo de la cultura científica. Parafraseando lo expuesto en los Estándares Básicos de Competencias en Ciencias Naturales (Mineducación, 2004), este documento define claramente lo que los estudiantes deben aprender definiendo un referente sobre lo que deben saber y saber hacer. Reconoce la importancia de desarrollar las competencias necesarias para la formación en Ciencias Naturales "desde la observación y la interacción con el entorno; la recogida de información... hasta la conceptualización, abstracción y uso de modelos explicativos...de los fenómenos observables...del universo" (p. 9).
Revisando las competencias de Ciencias Naturales de los diferentes grados de la Educación Básica Primaria, encontramos que los estudiantes deben comenzar a comparar movimientos y desplazamientos de seres vivos y objetos, para luego en sexto grado registrar observaciones y resultados mediante diagramas, gráficos y tablas, de manera que utilizan las matemáticas como herramienta para organizar, analizar y presentar los datos. Como se puede observar, los conceptos de cinemática aparecen en el currículo escolar a partir de cuarto grado, y luego se complementan con las matemáticas en la representación o interpretación de los datos mediante el uso de diversos registros como tablas, gráficos, fórmulas, entre otros.

Es en este contexto en el que se desarrolló esta investigación, donde se pretendía adoptar una vía, poco utilizada por los docentes en el trabajo de aula, que consistía en proporcionar al alumno una representación gráfica y a partir de ella, generar una serie de preguntas que exigían la comprensión de esta para poder interpretarla y así analizar lo que ocurría con la velocidad de los cuerpos en las gráficas Posición-Tiempo.

Se aclara que en este tipo de gráficos la velocidad termina siendo la pendiente de la línea recta asociada al movimiento rectilíneo uniforme que sigue el cuerpo. Se esperaba entonces generar un espacio de articulación de saberes que fuera coherente con el tipo de situaciones que se presentan en las pruebas estandarizadas a nivel nacional y romper con el esquema tradicional de enseñanza, que termina siendo una práctica reduccionista y operativa de las competencias científicas (Alvarado \& García, 2008).

\section{METODOLOGÍA}

Este proceso de investigación toma como población a todos los estudiantes matriculados para el año 2021 en los grados sexto a undécimo de una institución educativa privada ubicada en el área metropolitana de la ciudad de San José 
de Cúcuta. En total son $195(N=195)$ estudiantes y calculando el tamaño de la muestra $\left(n_{1}\right)$ a través de la Ecuación (1), y mediante la Ecuación (2) se determina un total de $76\left(n_{0}=76\right)$ estudiantes como tamaño óptimo de la muestra asumiendo una probabilidad de éxito del $70 \%(P=0.7)$, un error del $5 \%(e=0.05)$ y un nivel de confianza del $95 \%$ (equivalente a $Z=1.96$ ) con un nivel de significación del $5 \%(\alpha=0.05)$. Adicionalmente, se consideró como criterio de inclusión que los estudiantes asistieran a la institución de manera presencial, por lo que el proceso de muestreo utilizado fue probabilístico como lo señala Martínez (2012).

$$
\begin{gathered}
n_{1}=\frac{N * Z_{\alpha / 2}^{2} * P *(1-P)}{e^{2} *(N-1)+Z_{\alpha / 2}^{2} * P *(1-P)} \\
n_{0}=\frac{n_{1}}{1+{ }^{n_{1} / N}}
\end{gathered}
$$

En cuanto al instrumento, éste se compone de dos apartados, iniciando con el perfil de los estudiantes y luego proponiendo cinco situaciones derivadas del trabajo de investigación de Tejeda y Domínguez (2015), pero las preguntas se han ajustado a las características de los informantes y al contexto social en el que viven. El instrumento ha sido validado por el método de juicio de expertos, el cual estuvo conformado por el grupo de investigadores acompañados por el docente encargado de la asignatura en la institución, quien aportó su conocimiento del trabajo realizado y el lenguaje de los enunciados para asegurar la comprensión por parte de los estudiantes.

En cada situación propuesta, los estudiantes comenzaban seleccionando la respuesta correcta a elegir de entre un grupo de opciones (respuestas cerradas), pero luego se les pedía que justificaran el razonamiento de su elección. Los estudiantes disponían de un bloque continuo de 90 minutos, en el que primero se les sensibilizaba antes de rellenar el cuestionario.
Una vez recogidos los datos, se digitalizaron, representando cada fila las respuestas de un alumno. A continuación, se procesaron los datos organizando la información en tablas (simples o cruzadas) o gráficos para caracterizar el nivel exhibido por los estudiantes.

Por todo lo anterior, se concluye que esta investigación se ajusta a las características del enfoque cuantitativo a nivel descriptivo, ya que los investigadores acceden a los datos directamente de la fuente primaria, sin manipular ninguno de ellos, pero se procesan estadísticamente para destacar lo más relevante (Hernández-Sampieri \& Mendoza, 2018).

\section{RESULTADOS}

A continuación, se presentan los resultados derivados de la aplicación del instrumento utilizado en esta investigación, comenzando por el perfil demográfico de los informantes y analizando posteriormente los resultados obtenidos en cada una de las situaciones planteadas.

\section{PERFIL DEMOGRÁFICO Y ACADÉMICO}

En cuanto a las características de los informantes de esta investigación, se determinó que existe un predominio del género masculino con el $64.5 \%$ de los casos, en contraste con el $35.5 \%$ que corresponde a las mujeres. La edad oscila entre los 11 y los 17 años, con una media de 14.1 años y una desviación típica de 2.2 años, lo que nos permite calcular el coeficiente de variación, cuyo valor es de $15.6 \%$, que según Amón (2000) ofrece una variación admisible. El coeficiente de asimetría es de -0.119 , por lo que el histograma de los datos tiene una ligera cola hacia la izquierda con frecuencias distribuidas en todo el rango, generando un efecto de distribución platicúrtica que es coherente con el valor de la curtosis (-1.457). Estos dos estadísticos caracterizan la diversidad de edades observada en los informantes según los grados analizados. 
Con respecto al grado de los informantes, se identificó que participaron desde sexto hasta undécimogradocon porcentajesqueoscilan entre el $10.5 \%$ y el $22.4 \%$, con un menor porcentaje en octavo grado y una mayor presencia en sexto. Al desagregar la concentración de género por grado, se utilizó el uso de tablas cruzadas, lo que permitió determinar que las mujeres se concentran en los grados once, nueve y siete, mientras que los hombres predominan en los grados seis y diez. En octavo grado, hay una distribución casi igual por género.

Cuando se les preguntó si habían visto materias de física en la institución, todos los estudiantes respondieron que sí, lo cual es coherente con el énfasis de la institución educativa, que corresponde a la formación en ciencias naturales. Para concluir este acercamiento al conocimiento del proceso académico, se preguntó a los estudiantes si les gustaban las clases de Física y la forma en que el docente orienta la asignatura, y se determinó que el $38.2 \%$ no estaba satisfecho con este aspecto, mientras que el porcentaje restante (equivalente al $61.8 \%$ ) dijo que las clases eran buenas y que tenía una actitud positiva hacia la asignatura. Este aspecto coincide con lo señalado por Fernández et al. (2009), quienes afirman que las prácticas tradicionales de enseñanza centradas en la repetición, la memoria y el docente deben ser sustituidas por procesos de enseñanza centrados en el alumno, dándole la oportunidad de asumir un papel activo y comprometido en su propio aprendizaje.

\section{SITUACIONES ASOCIADAS A LA CINEMÁTICA}

A continuación, se presentan los resultados derivados de cada una de las situaciones propuestas en relación con la interpretación de los gráficos Posición-Tiempo. Como se destaca en Prada-Núñez et al. (2016), es necesario articular diversos registros de representación semiótica para resolver las debilidades conceptuales que tienen los estudiantes en relación con los temas.

Situación 1. Se presenta una gráfica PosiciónTiempo en la que hay dos objetos $A$ y $B$, donde el objeto A parte de la posición de referencia (cero) y en el transcurso de 5 segundos alcanza los 50 metros, mientras que el objeto $B$ parte de una posición 20 metros más alejada del punto de referencia y durante los mismos 5 segundos se desplaza hasta una posición de 35 metros. El gráfico muestra que las rectas se cruzan 3 segundos después del inicio del desplazamiento simultáneo de ambos objetos, por lo que se identifican dos tramos clave en el gráfico, antes y después de los primeros 3 segundos.

Cuando se preguntó a los estudiantes si podían inferir del gráfico si en algún momento los objetos $\mathrm{A}$ y $\mathrm{B}$ tendrían la misma velocidad, el $61.8 \%$ (equivalente a 47 informantes) dijo que sí, mientras que el $38.2 \%$ restante (equivalente a 29 informantes) dijo que no. Los argumentos dados se muestran en la Tabla 1 , donde se pueden observar varios criterios para apoyar el argumento. Los argumentos dados se muestran en la Tabla 1, donde se pueden observar diversos criterios para apoyar la argumentación, por ejemplo, el $83.0 \%$ de los que dijeron que en algún momento los objetos tuvieron la misma velocidad basaron su argumento en el punto de corte de ambas líneas donde se asume la igualdad de valores en el mismo, coincidiendo con las conclusiones de la investigación de Martínez y Iturriza (2016), pero de ellos sólo el $23.4 \%$ reconoce que la pendiente de la línea corresponde a la velocidad, mientras que los demás asocian el cruce de líneas en función de una de las variables asociadas a cada eje. Cabe destacar que existe un argumento común entre los estudiantes que aceptan y los que rechazan la igualdad de la velocidad, basado en el ángulo de inclinación de la recta con respecto al eje de abscisas. Por otro lado, el $26.7 \%$ de los que contestaron que no, aseguran su argumento en 
función de la posición inicial de ambos cuerpos. Por último, cabe destacar que aproximadamente el $8 \%$ del total de los informantes (equivalente al $20.7 \%$ de los que dijeron que no) afirmaron no entender la situación propuesta

Tabla 1. Cuadro comparativo de los argumentos dados según la opción de respuesta.

\begin{tabular}{|l|c|c|}
\hline \multicolumn{1}{|c|}{ Argumentos } & Aceptar & Rechazar \\
\hline $\begin{array}{l}\text { Alcanzan la misma velocidad (la pendiente) cuando las dos rectas se } \\
\text { cruzan. }\end{array}$ & $23.4 \%$ & \\
\hline Porque se cruzan al llegar a 30 centímetros. & $25.5 \%$ & \\
\hline $\begin{array}{l}\text { Porque en el tiempo 3 segundos ambos objetos tienen la misma } \\
\text { velocidad. }\end{array}$ & $34.1 \%$ & \\
\hline Porque la línea A tiene más pendiente que la línea B, va más rápido. & $17.0 \%$ & $51.7 \%$ \\
\hline La posición inicial de las líneas es diferente por lo que no son iguales. & & $27.6 \%$ \\
\hline No entiendo la situación & $100.0 \%$ & $20.7 \%$ \\
\hline \multicolumn{1}{|c|}{ Total } & $100.0 \%$ \\
\hline
\end{tabular}

Situación 2. De nuevo se muestra una gráfica Posición-Tiempo que corresponde al recorrido que sigue un coche en línea recta suponiendo una gasolinera como punto de referencia, donde se identifican tres tramos con características diferentes: a) el coche se sitúa 10 metros antes de la gasolinera (valor negativo) y en un segundo alcanza la posición 10 metros después de la gasolinera; b) luego se detiene en este lugar durante dos segundos; c) posteriormente avanza 10 metros más durante tres segundos. $A$ partir de la descripción, se identifica que hay un valor diferente de velocidad en cada trayectoria, siendo la primera trayectoria (de cero a un segundo) la que tiene mayor velocidad. A partir de este razonamiento, los estudiantes debían seleccionar el intervalo de tiempo en el que se observaba la mayor velocidad. De la Tabla 2 se desprende que el $30.3 \%$ argumenta que la velocidad máxima se alcanza en el primer recorrido porque se tarda menos tiempo o porque se ve que sube más rápido en la recta. El $47.4 \%$ de los informantes eligió el recorrido 3 porque es el recorrido en el que se alcanza la posición máxima, sin saber el tiempo que tarda en llegar, es decir, validan su argumento sólo observando los valores del eje de ordenadas.

Tabla 2. Tabla de frecuencias de las rutas seguidas.

\begin{tabular}{|l|c|c|}
\hline \multicolumn{1}{|c|}{ Opciones de respuesta } & Frecuencia & Porcentaje \\
\hline Trayecto 1: entre cero y un segundo & 23 & 30.3 \\
\hline Trayecto 2: entre uno y tres segundos & 13 & 17.1 \\
\hline Trayecto 3: entre tres y seis segundos & 36 & 47.4 \\
\hline La velocidad es la misma durante todo el trayecto & 4 & 5.3 \\
\hline \multicolumn{1}{|c|}{ Total } & 76 & 100.0 \\
\hline
\end{tabular}


De forma complementaria, se pregunta a los estudiantes, si se quiere que la velocidad del coche sea de 10 metros por segundo en el viaje tres, ¿cuál de las siguientes opciones sería la correcta? La respuesta correcta que indica que se alcanza una posición final de 40 metros en los mismos tres segundos fue proporcionada por el $18.4 \%$ de los informantes, mientras que el porcentaje restante optó por opciones incompletas o engañosas como aumentar 10 metros más desde la posición inicial de este recorrido o aumentar a 30 metros en los tres segundos. Esta última respuesta demuestra que tienen el concepto de velocidad como cociente entre posición y tiempo, pero desconocen la posición inicial del objeto.

Situación 3. En esta situación, se dan a los estudiantes dos gráficas, una de PosiciónTiempo que muestra un aumento de posición de 10 metros por segundo desde el origen, mientras que la segunda gráfica de Velocidad-Tiempo muestra una función constante con un valor de 10 metros por segundo durante el tiempo de observación. Los estudiantes debían responder si las gráficas correspondían al movimiento del mismo objeto. A partir de la primera gráfica se puede determinar la velocidad y comprobar que es la misma en todo momento, por lo que es correcto afirmar que la velocidad es constante, por lo que se concluye que las dos gráficas están asociadas y que se corresponde con el comportamiento de la posición y la velocidad en el tiempo.

Se determinó que el $28.9 \%$ dice que las dos gráficas corresponden al movimiento de un mismo objeto, argumentando que, a mayor posición, mayor tiempo, lo que demuestra que no tienen claros los conceptos de variable independiente y dependiente. De este grupo, un pequeño grupo de estudiantes mencionó que las gráficas siempre avanzan en el tiempo y por lo tanto corresponden al mismo movimiento, por lo que se evidencia en ellas el tiempo como variable independiente, pero con total desconocimiento del concepto de velocidad como la pendiente de la línea recta en la gráfica Posición-Tiempo.

Aproximadamente el $71.1 \%$ de los informantes afirma que las gráficas no corresponden al mismo movimiento, apoyándose en dos argumentos: a) el $37.0 \%$ afirma que "el enunciado dice que es una función del tiempo, a pesar de ser el mismo tiempo las gráficas son diferentes, por lo que no son iguales", lo que demuestra que desconocen la relación entre el tiempo y las demás variables; b) el $34.0 \%$ restante afirma que "una función es creciente y la otra es una constante, por lo que no son iguales". Este argumento demuestra que reconocen algunas funciones reales desde el punto de vista matemático, pero desconocen por completo el contexto físico de las variables en cuestión.

Situación 4. Se menciona en el enunciado que un cuerpo se deja caer libremente desde cierta altura y, por tanto, está sometido únicamente a la acción de la aceleración de la gravedad. Se pidió a los estudiantes que seleccionaran, entre cuatro gráficas Posición-Tiempo diferentes, la que correspondía al movimiento del cuerpo. La tabla 3 muestra que sólo el $13.2 \%$ de los informantes seleccionó la respuesta correcta (opción 1) que representa el efecto de la aceleración de forma exponencial en la que aumenta su tasa de incremento. Del porcentaje restante, el 35.8\% (opción 4) tiene dificultades conceptuales a nivel matemático dado que la gráfica no representa una función, mientras que el $31.6 \%$ (opción 3) asocia la gráfica con la posible trayectoria que sigue un cuerpo cuando sigue un tiro parabólico, asociándola posiblemente con la caída del cuerpo. Finalmente, el $18.4 \%$ que seleccionó la opción 2, asume un movimiento uniforme que va en contra de la presencia de la aceleración de la gravedad, evidenciando así debilidades en los conceptos de cinemática. 
Tabla 3. Descripción del tipo de gráfico Posición-Tiempo que sigue el movimiento del cuerpo al caer.

\begin{tabular}{|l|c|c|}
\hline \multicolumn{1}{|c|}{ Opciones de respuesta } & Frecuencia & Porcentaje \\
\hline $\begin{array}{l}\text { Opción 1. Función cuadrática creciente que parte del origen y alcanza } \\
\text { una posición de 40 cm en dos segundos. }\end{array}$ & 10 & 13.2 \\
\hline $\begin{array}{l}\text { Opción 2. Función lineal creciente a razón de 10 metros por segundo } \\
\text { partiendo del origen. }\end{array}$ & 14 & 18.4 \\
\hline $\begin{array}{l}\text { Opción 3. Función cuadrática decreciente con vértice en el punto } \\
(0.40) \text { y que llega al suelo en dos segundos. }\end{array}$ & 24 & 31.6 \\
\hline $\begin{array}{l}\text { Opción 4. Recta vertical paralela al eje y que, con un tiempo constante } \\
\text { de dos segundos, desciende de 40 a 0 centímetros. }\end{array}$ & 28 & 36.8 \\
\hline Total & 76 & 100.0 \\
\hline
\end{tabular}

Situación 5. La prueba termina con una representación gráfica en la que hay dos líneas rectas paralelas que representan la posición de dos cuerpos que se mueven a una velocidad constante de 10 kilómetros por hora durante seis horas cada uno, con la diferencia de que el cuerpo A sale tres horas antes que el cuerpo B. Se pidió a los estudiantes que dedujeran del gráfico la velocidad de los dos cuerpos.

Aproximadamente el $63.2 \%$ de los informantes afirma que uno de los cuerpos tiene mayor velocidad, por ejemplo, el $39.5 \%$ dice que la velocidad del cuerpo $A$ es mayor porque recorrió la distancia en menos tiempo mientras que el $23.7 \%$ afirma que el cuerpo $B$ es el más rápido a pesar de haber llegado el último. Estas respuestas muestran el análisis de las variables por separado y no como una función en la que se establece una relación de correspondencia entre los valores de dos variables.

Aproximadamente el $13.2 \%$ de los encuestados afirman que la velocidad aumenta con el paso del tiempo independientemente del cuerpo observado, pero no se atreven a afirmar que la velocidad es la misma, demostrando que desconocen que si dos rectas son paralelas $\left(Y_{1}\right.$ paralela a $Y_{2}$ ), esto implica la igualdad de sus pendientes $\left(m_{1}=m_{2}\right)$ tal y como se recoge en el sistema de ecuaciones lineales canónicas (3), por lo que cuando se cumple esta condición, el aumento es el mismo en ambos casos. Los valores $B_{10}$ y $B_{20}$ son los interceptos con el eje de ordenadas y $X$ la variable independiente.

$$
\left\{\begin{array}{l}
Y_{1}=m_{1} x+B_{10} \\
Y_{2}=m_{2} x+B_{20}
\end{array}\right.
$$

Se evidencian mayores dificultades a la hora de extraer los datos de la gráfica para poder determinar las velocidades. Finalmente, el $23.7 \%$ dice que los dos cuerpos tienen la misma velocidad a pesar de que se desplazan con diferente diferencia de tiempo. El 4.9\% dice que tienen la misma velocidad, sólo que tienen posiciones diferentes, respuesta que muestra confusión entre las variables asociadas a cada eje de coordenadas.

Como síntesis del proceso adelantado, se puede decir que es necesario que el docente en el desarrollo de las clases promueva la articulación coherente de los diferentes registros de representación, tal como se esboza en los hallazgos del trabajo de Choco (2019), en el que se afirma que parte de las dificultades se deben al confinamiento a un solo registro de representación, es decir, que el docente en su proceso de enseñanza siempre orienta su trabajo a ir de la expresión algebraica a la representación en el plano cartesiano, pasando por el registro tabular, pero cuando se intenta el proceso inverso, automáticamente surgen una serie de obstáculos en los estudiantes. 


\section{CONCLUSIONES}

Luego de realizar esta investigación, se determinó que a pesar de ser una institución educativa cuyo énfasis es la formación en Ciencias Naturales, donde los estudiantes comienzan a trabajar con conceptos básicos de Física, como distancia, velocidad, fuerza, reposo, movimiento, entre otros, desde los grados de Educación Básica Primaria.

En el caso particular de los estudiantes de cualquiera de los grados de la Secundaria Básica o Técnica, comienzan a analizar intuitivamente el movimiento de los cuerpos, como una relación entre la distancia recorrida por un cuerpo a medida que transcurre el tiempo, para luego pasar a interpretar y describir lo que sucede en las gráficas Posición-Tiempo. De lo anterior, se puede concluir que, con respecto a la propuesta curricular de la institución, se esperaría que los estudiantes sean capaces de resolver las situaciones propuestas, lo que genera un punto de reflexión sobre cómo el docente de Física está desarrollando los contenidos y competencias que está promoviendo en el trabajo de aula.

Además, se determinó que a lo sumo uno de cada cuatro informantes fue eficiente en la articulación de conceptos matemáticos aplicados a situaciones o planteamientos propios de la Física. Este aspecto reduce la probabilidad de éxito en pruebas como la Saber 11, dado que muchos de los planteamientos se basan en el registro gráfico y requieren la capacidad de abstraer datos para resolver situaciones problemáticas.

\section{REFERENCIAS BIBLIOGRÁFICAS}

Alvarado, L., \& García, M. (2008). Características más relevantes del paradigma sociocrítico: su aplicación en investigaciones de educación ambiental y de enseñanza de las ciencias realizadas en el Doctorado de Educación del Instituto Pedagógico de Caracas. Sapiens: Revista Universitaria de Investigación, 9(2), 187-202.

Amón, J. (2000). Estadística para psicólogos II. Probabilidad. Estadística inferencial (9a. ed.). Madrid: Pirámide.

Castro, A., \& Ramírez, R. (2013). Enseñanza de las ciencias naturales para el desarrollo de competencias científicas. Amazonia Investiga, 2(3), 30-53.

Choco, A. F. (2019). Dificultades y errores de estudiantes de grado undécimo en torno al estudio de las funciones racionales (tesis de pregrado). Universidad del Valle, Valle del Cauca, Colombia.

Congreso de la República de Colombia. (1994, 08 de febrero). Ley 115 de 1994. Por la cual se expide la ley general de educación. https://www.mineducacion.gov.co/1621/ articles-85906 archivo pdf.pdf

Domínguez, M. R. (2009). La importancia de la comunicación no verbal en el desarrollo cultural de las sociedades. Razón y palabra, 14(70), 1-28.

Enkvist, I. (2010). El éxito educativo finlandés Bordón. Revista de pedagogía, 62(3), 4967.

Fernández, M. T., Tuset, A. M., Pérez, R. E., \& Leyva, A. C. (2009). Concepciones de los maestros sobre la enseñanza y el aprendizaje y sus prácticas educativas en clases de ciencias naturales. Enseñanza 
de las ciencias: revista de investigación y experiencias didácticas, 27(2), 287-98.

Hernández-Sampieri, R., \& Mendoza, C. (2018). Metodología de la investigación (Vol. 4). México D. F.: McGraw-Hill.

Martínez, C. (2012). Estadística y muestreo (13a . ed). Bogotá: Ecoe Ediciones.

Martínez, J., \& Iturriza, H. (2016). La Resolución de problemas como recurso instruccional para la enseñanza de la Cinemática en el contexto rural. Revista Multidisciplinaria Dialógica, 13(1), 149-179.

Ministerio de Educación Nacional. (1998). Lineamientos Curriculares de Ciencias Naturales y Educación Ambiental. Bogotá: Mineducación.

Ministerio de Educación Nacional. (2004). Estándares Básicos de Competencias en Ciencias Naturales y Ciencias Sociales. Bogotá: Mineducación.

Prada-Núñez, R., Hernández-Suárez, C., Ramírez-Leal, P. (2016). Comprensión de la noción de función y la articulación de los registros semióticos que la representan entre estudiantes que ingresan a un programa de ingeniería. Revista Científica, 2(25), 188-205.

Tejeda, S., \& Domínguez, A. (2015). Dificultades conceptuales en la relación de gráficas de cinemática: Estudio de casos. LatinAmerican Journal of Physics Education, 9(S1), 1-6.

Velez, R. M., Ponce, C. V., \& Solórzano, S. E. (2016). Importancia de la comunicación en el aprendizaje. SATHIR, (11), 252-260. 